\title{
Intravascular Ultrasound Image Segmentation: A Fast-Marching Method
}

\author{
Marie-Hélène Roy Cardinal ${ }^{1}$, Jean Meunier ${ }^{2}$, Gilles Soulez $^{3}$, Éric Thérasse $^{3}$, \\ and Guy Cloutier ${ }^{1}$ \\ 1 Laboratory of Biorheology and Medical Ultrasonics, University of Montreal \\ Hospital. \\ roycarmh@iro.umontreal.ca, guy.cloutier@umontreal.ca \\ 2 Département d'Informatique et de Recherche Opérationnelle, University of \\ Montreal. \\ meunier@iro.umontreal.ca \\ 3 Radiology, Radio-Oncology and Nuclear Medicine Department, University of \\ Montreal Hospital.
}

\begin{abstract}
Intravascular ultrasound (IVUS) is a medical imaging technique that not only provides three-dimensional information about the blood vessel lumen and wall, but also directly depicts atherosclerotic plaque structure and morphology. Automatic processing of large data sets of IVUS data represents an important challenge due to ultrasound speckle and technology artifacts. A new semi-automatic IVUS segmentation model, the fast-marching method, based on grayscale statistics of the images, is compared to active contour segmentation. With fast-marching segmentation, the lumen, intima plus plaque structure, and media contours are computed in parallel. Preliminary results of this new IVUS segmentation model agree very well with vessel wall contours. Moreover, fast-marching segmentation is less sensitive to initialization with average distance between segmentation performed with different initializations $<0.85 \%$ and Haussdorf distance $<2.6 \%$.
\end{abstract}

\section{Introduction}

Over the past few years, the intravascular ultrasound (IVUS) technology has become very useful in research and in some clinical applications, particularly for studying atherosclerotic disease. IVUS is a catheter based medical imaging technique that produces cross-sectional images of blood vessels. It also provides quantitative assessment of the vascular wall, information about the nature of atherosclerosis lesions as well as the plaque shape and size. IVUS adds complementary information to angiography when characterizing atherosclerotic plaque [13. The tomographic nature of intravascular ultrasound also makes 3D reconstruction of the vessel wall possible. Furthermore, quantitative measurements of atherosclerosis disease such as plaque volume, intima-media thickness, vascular remodeling and lumen area stenosis can be retrieved from IVUS [12. However, the amount of data produced by an intravascular ultrasound acquisition can be 
quite large, making the analysis of the data long and fastidious. Automatic image segmentation should ease the examination of intravascular ultrasound series. On the other hand, because IVUS image quality remains poor due to speckle noise, imaging artifacts and shadowing of parts of the vessel wall by plaque components like calcifications, it is necessary to develop specific segmentation methods.

So far, a number of segmentation techniques have been developed for IVUS data analysis. A great portion of this work is based on local properties of image pixels: gradient based region growing [2], gradient active surfaces [7, pixel intensity and gradient active contours 8], graph search using first and second derivative gradient filters combined to local grayscale properties [3,9] or Sobel-like edge operator [19, and multiagent image segmentation 11. Texture information has also been investigated as a basis for IVUS segmentation [14. Finally, optimization of a MAP estimator modeling ultrasound speckle and contour geometry was considered [5]. Still, in 2001, a clinical expert consensus from the American College of Cardiology [12] reported that no IVUS edge detection method had found widespread acceptance by clinicians. In the present study, usage of a global image characteristic, i.e. the probability density functions of the IVUS wall components, combined to a fast-marching segmentation model, a regionbased method handling topological contour changes, is proposed to address the IVUS segmentation problem.

\section{Segmentation Model}

\subsection{Fast-Marching Method}

The fast-marching method is a particular case of the level-set approach introduced by Osher and Sethian to follow interface propagation [15[16]. The level-set model defines the interface as the zero level of a function $\phi$ of higher dimension. The $\phi$ function moves in its normal direction according to a speed function $F$. For a $2 \mathrm{D}$ interface, the evolution of $\phi$ is given by Eq. (1) with initial surface $\phi(x, y, t=0)$.

$$
\frac{\partial \phi(x, y, t)}{\partial t}+F|\nabla \phi|=0 .
$$

The level-set model can be applied to image segmentation by interpreting image contours as the propagating interface final position [10]16]. To achieve this, the speed function should become close to zero when the propagating front meets with the desired contour thus making the interface stop at this position and then ending the segmentation process.

Fast-marching consists of the evolution of an interface propagating under a unidirectional speed function. In this case, the evolving contour must be inside the region to segment (for a positive speed function or outside for a negative one). In the fast-marching formulation, the evolution of the contour is expressed in terms of the arrival time $T(x, y)$ of the contour at point $(x, y)$. The $T$ function satisfies Eq. (22), stating that the arrival time difference between two adjacent pixels increases as the velocity of the contour decreases. 


$$
|\nabla T| F=1
$$

The propagation of the interface is done via the construction of the arrival time function. The construction algorithm [17] selects the interface point having the smallest arrival time and calculates the arrival times of its neighbors, and so on until the interface has propagated across the whole image. The interface front is considered as stationary when the time gradient is sufficiently high.

Since multiple contours (lumen, intima and media) must be identified on the IVUS series, image segmentation is done via a multiple interface extension of the fast-marching algorithm [18. A speed function is then defined for each propagating interface. Also, every interface associated to the vessel wall contours evolves at a velocity defined in terms of the probability density function $P_{l \in L}$ of the corresponding anatomical structure. The propagation speed of interface $m \in L$, where $L$ is the set $1,2, \ldots, N$ of the $N$ evolving interfaces, is given by Eq. (3).

$$
F_{m}(x, y)=\left(1+\frac{1}{N_{\nu}} \sum_{s \in \nu} \frac{\sum_{l \neq m, l \in \mathrm{E}} P_{l}\left(I_{s}\right)}{P_{m}\left(I_{s}\right)}\right)^{-1}
$$

Where $I_{s}$ is the grayscale of pixel $s$ at position $(x, y)$ in image $I, P_{l}\left(I_{s}\right)$ and $P_{m}\left(I_{s}\right)$ are the measured occurring probabilities of pixel $I_{s}$ in region $l$ and $m$. Because the occurring probability is more significant for a region than for a single pixel, the speed function is calculated over a certain number $N_{v}$ of neighbors, which are the 8-connected pixels around $(x, y)$. According to Eq. (3), the $m$ interface velocity will always be positive and take higher values when it is inside a region having a grayscale distribution close to $P_{m}$. This velocity function was chosen because of its general form (no specific PDF is required) and because it provides neighborhood averaging.

To allow comparison, the segmentation model has also been implemented using a gradient based speed function of the form $F(x, y)=1 /(1+|\nabla I(x, y)|)$. Notice that the multiple interface fast-marching enables simultaneous segmentation of different parts of the vessel wall.

\subsection{Active Contours Model}

To compare the new IVUS segmentation method based on the fast-marching, active contours or snakes, introduced by Kass et al. 6] have also been implemented using PDFs and image gradient. The snake general energy function is given by Eq. (41). For PDFs based snakes [11, the external energy term satisfies Eq. (17).

$$
\begin{aligned}
E_{\text {snake }}(V)= & \sum_{i=1}^{n} \alpha E_{d \_m e a n}\left(v_{i}\right)+\beta E_{\text {curv }}\left(v_{i}\right)+\gamma E_{\text {ext }}\left(v_{i}, I\right) . \\
& E_{d \_m e a n}\left(v_{i}\right)=|d-| v_{i}-v_{i-1}|| .
\end{aligned}
$$




$$
\begin{gathered}
E_{\text {curv }}\left(v_{i}\right)=\left|v_{i-1}-2 v_{i}+v_{i+1}\right| . \\
E_{\text {ext }}\left(v_{i}, I\right)=-\frac{1}{N_{\text {in }}} \sum_{s \in v_{i}^{\text {in }}} \ln P_{\text {in }}\left(I_{s}\right)-\frac{1}{N_{\text {out }}} \sum_{s \in v_{i}^{\text {out }}} \ln P_{\text {out }}\left(I_{s}\right) .
\end{gathered}
$$

Where $V$ is the set of $n$ points $v_{i}$ making the contour, $\alpha, \beta$ and $\gamma$ are weighting factors, $I_{s}$ is the intensity of pixel $s, E_{d \_m e a n}$ is an energy term keeping distance between neighboring points close to the mean distance $d, E_{\text {curv }}$ is the energy term minimizing the curvature, $P_{\text {in }}$ and $P_{\text {out }}$ are the PDFs inside and outside the contour. $E_{\text {ext }}$ is the energy term related to image $I$ 's characteristics. The energy function is calculated over regions $v_{i}^{\text {in }}$ and $v_{i}^{\text {out }}$. These regions are delimited by the current point, the two closest contour points and these three points extended by five pixels inside and outside the contour along its normal direction. For the gradient implementation of snakes [7, the external energy function is given by

$$
E_{\text {ext }}\left(v_{i}, I\right)=\left(g_{\min }\left(v_{i}\right)-g\left(v_{i}\right)\right) /\left(g_{\max }\left(v_{i}\right)-g_{\min }\left(v_{i}\right)\right)
$$

where $g$ is the gradient, $g_{\min }$ and $g_{\max }$ are the minimum and maximum values of gradient among the $v_{i}$ neighbors. Segmentation is finished when minimal energy is reached. The energy function is minimized by evaluating iteratively the energy for three contour point neighbors in normal direction, inside and outside the snake. Neighbors minimizing $E_{\text {snake }}(V)$ are chosen to replace contour points of higher energy. Segmentations of the lumen, intima plus plaque structure and media are performed one at a time.

\subsection{Data Initialization}

The fast-marching segmentation requires an initialization which was done by the user on longitudinal images of the IVUS series. Contours were manually traced on 3 to 4 different cut planes, creating a set of contour points on each IVUS frame. Then, splines passing through these points were computed and used as initial contours, directly for the active contours model and slightly shrunk for the fast-marching algorithm (because the propagating interface must be inside the region to segment).

\subsection{Probability Density Function Estimation}

For intravascular ultrasound images, the lumen, intima plus plaque structure, and media PDFs can be modeled by a shifted Rayleigh law 44. The PDF, with parameters $\{\min , a\}$, is given by

$$
R(u ; \min , a)=\frac{u-\min }{a^{2}} \exp \left(-\frac{(u-\min )^{2}}{2 a^{2}}\right)
$$

with $u>\min , a>0$, and the variance $=a^{2}(4-\pi) / 2$. By measuring the standard deviation and minimum gray level value of each area outlined by the user's initialization, the parameters $\{\min , a\}$ were estimated for each region of interest. 


\subsection{Materials}

The active contours and the multiple interface fast-marching methods were used to segment the lumen, intima plus atherosclerotic plaque structure, and media of a 200 IVUS image series of a femoral artery. Data were acquired with a Jomed equipment (In-vision gold, Helsingborg Sweden) using a $20 \mathrm{MHz}$ transducer. Images of size $10 \times 10 \mathrm{~mm}$ were digitized on 384 x 384 pixel matrices and stored using the Dicom standard. The acquisition was done at a 10 images/sec frame rate and the catheter pullback velocity was set to $1 \mathrm{~mm} / \mathrm{sec}$ generating $0.1 \mathrm{~mm}$ thick slices. Image acquisition was not ECG-gated.

\section{Results}

In most cases, a qualitative analysis of the PDFs based fast-marching segmentation revealed detected contours that were very close to the lumen, intima and media boundaries. A typical segmentation result is shown in Fig. 1.

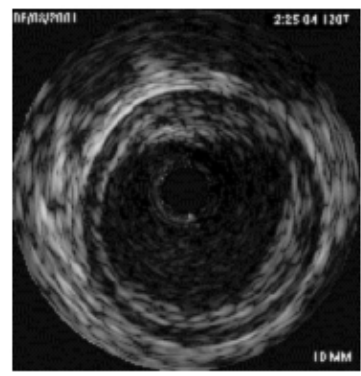

(a)

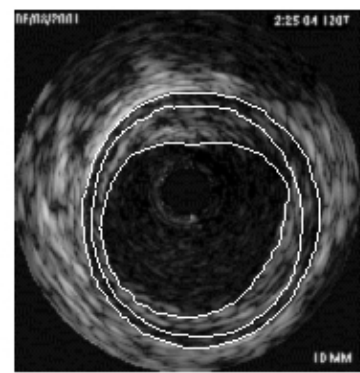

(b)

Fig. 1. (a) Typical IVUS cross-sectional image and (b) IVUS image with PDFs based fast-marching detected contours of lumen, intima plus plaque and media.

Fig. 2 and Fig. 3 show a volumic representation of the lumen and media contours also obtained with PDFs based fast-marching segmentation. On each figure, 20 of the 200 set of detected contours are drawn over two longitudinal cut planes of the image series. The other methods (fast-marching with gradient and active contour with both gradient and PDFs) also provided similar qualitative results (data not shown).

To quantify the variability of the segmentation under different initializations, fast-marching and active contour methods, both based on gradient and PDFs, were applied to a 15-image sequence with three different sets of initial contours. Table 1 shows the average and Haussdorf distances (maximum distance to the closest point) between resulting contours. The distances are presented in percentage of the image size. 


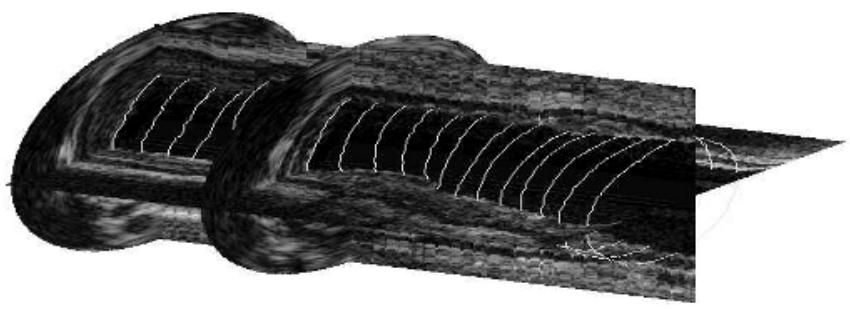

Fig. 2. Lumen contours from PDFs based fast-marching segmentation displayed over two IVUS images and two longitudinal cuts.

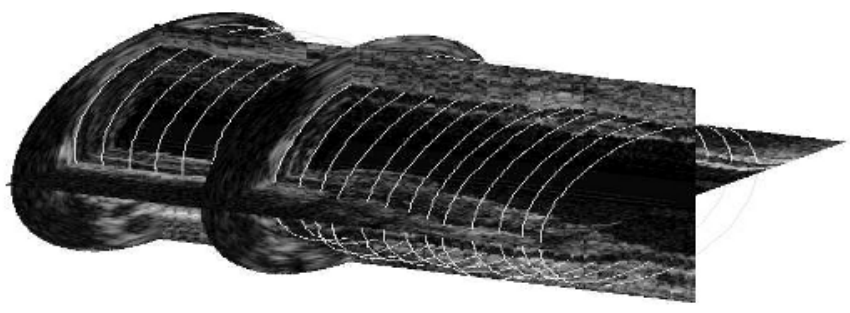

Fig. 3. Media contours from PDFs based fast-marching segmentation displayed over two IVUS images and two longitudinal cuts.

\section{Discussion}

The goal of this work was to demonstrate the IVUS segmentation potential of the fast-marching method, and the usefulness of region statistics such as probability density functions of vessel wall anatomical structures. Fig. 1 showed that vessel wall boundaries can be identified even when the contrast is not very high and when the shape is irregular as for the luminal contour. Figs. 2 and 3 demonstrated that segmentation results followed the desired boundaries for a whole volume. In the case of the snake model, the boundaries seemed to be more affected when they were not well defined (results not shown).

Table 1. Average distance (AD) and Haussdorf distance (HD) between contours with different initializations in percent.

\begin{tabular}{ll|cc|cc|cc}
\hline \multirow{2}{*}{ AD and HD in $\%$} & \multicolumn{2}{|c|}{ Lumen } & \multicolumn{2}{c|}{ Intima } & \multicolumn{2}{c}{ Media } \\
& & AD & HD & AD & HD & AD & HD \\
\hline \hline Fast- & Gradient & 0.86 & 2.90 & 0.88 & 3.14 & 0.60 & 2.65 \\
Marching & PDFs & 0.74 & 2.13 & 0.84 & 2.57 & 0.61 & 2.35 \\
\hline Active- & Gradient & 0.93 & 3.33 & 1.07 & 3.98 & 0.65 & 3.27 \\
Contour & PDFs & 0.63 & 3.66 & 0.78 & 4.44 & 0.42 & 2.47 \\
\hline
\end{tabular}


Qualitative analysis of the detected contours indicates that the fast-marching is an accurate segmentation method for intravascular ultrasound imaging, but this should be further confirmed from validation with several manually traced contours by independent experts. This step would also enable quantitative accuracy comparison of active contour and evolving interface algorithms. Nevertheless, quantitative evaluation and comparison of the variability or precision of active contours and fast-marching under different initializations was performed in Table 1. The average and Haussdorf distance comparison metrics were chosen instead of area or perimeter differences because they directly depict point to point contour variations. Table 1 indicates that PDFs based fast-marching has the smallest Haussdorf distance, which remained under $2.6 \%$ for all boundaries, with a relatively small average distance between contours, of less than $0.84 \%$. Thus, fast-marching detected contours had small variations when initialized differently and the maximum distance to the closest point, representing the worst case, stayed low. PDFs active contours also had a small average distance of less than $0.78 \%$, but the worst variation could be higher than for the evolving interfaces. Table 1 also showed that gradient based segmentations, independently of the method, produced more variations (less precision) than the ones using grayscale PDFs. This indicates that the PDF information seems more constant and possibly more reliable over the whole image than the gradient.

These preliminary segmentation results showed that the fast-marching approach is a promising technique for IVUS image processing. In the near future, a need would be to automate the initialization step to minimize user's interactions by fitting a mixture of PDFs directly to the IVUS image, and then by roughly estimating an initial segmentation. This way it is hoped that the PDFs based fast-marching algorithm may become a reliable fully automatic segmentation tool of choice for IVUS.

Acknowledgments. This work was supported by grants from the Canadian Institute of Health Research (G.C., G.S., \#MOP-53244) and ValorisationRecherche Québec (group grant \#2200-094), and by research scholarships from the Fonds de la Recherche en Santé du Québec (G.C. and G.S.).

\section{References}

1. Bovenkamp, E.G.P., Dijkstra, J., Bosch, J.G., Reiber, J.H.C.: Multiagent IVUS image interpretation. SPIE Proceedings: Medical Imaging 2003: Image Processing, San-Diego, California, USA, vol 5032, 2003, pages 619-630.

2. Brathwaite, P.A., Chandran, K.B., McPherson, D.D., Dove, E.L.: Lumen Detection in Human IVUS Images Using Region-Growing. IEEE Computers in Cardiology, pages 37-40, 1996.

3. Dijkstra, J., Koning, G., Reiber, L. H. C.: Quantitative measurements in IVUS images. Int J Cardiac Imag, vol. 15, pages 513-522, 1999.

4. Goodman, J.W.: Some fundamental properties of speckle. J Opt Soc Am, vol. 66 (11), pages 1145-1150, 1976. 
5. Haas, C., Ermert, H., Holt, S., Grewe, P., Machraoui, A., Barmeyer, J.: Segmentation of 3D Intravascular Ultrasonic Images based on a Random Field Model. Ultrasound Med Biol, vol. 26 (2), pages 297-306, 2000.

6. Kass, M., Witkin, A., Terzopoulos, D.: Snakes: Active contour models. International Journal of Computer Vision, vol. 1 (4), pages 321-331, 1988.

7. Klingensmith, J. D., Shekhar, R., Vince, G.: Identification of Luminal and MedialAdventitial Borders in IVUS Images. IEEE T Med Imaging vol. 19 (10), 2000.

8. Kovalski, G., Beyar, R., Shofti, R., Azhari, H.: Three-Dimensional Automatic Quantitative Analysis of Intravascular Ultrasound Images. Ultrasound Med Biol, vol. 26 (4), pages 527-537, 2000.

9. Koning, G., Dijkstra, J., von Birgelen, C., Tuinenburg, J. C., Brunette, J., Tardif, J.-C., Oemrawsingh, P. W., Sieling, C., Melsa, S., Reiber, L. H. C.: Advanced contour detection for three-dimensional intracoronary ultrasound: a validation in vitro and in vivo. Int J Cardiac Imag, vol. 18, pages 235-248, 2002.

10. Malladi, R., Sethian, J.A. Vemuri, B.C.: Shape Modeling with Front Propagation: A Level Set Approach. IEEE T Pattern Anal, 17 (2), pages 158-175, 1995.

11. Mignotte M., Meunier J.: A multiscale optimization approach for the dynamic contour-based boundary detection issue. Comput Med Imag Graph, vol. 25 (3), pages 265-275, 2001.

12. Mintz, G.S., Nissen, S.E., Anderson, W.D., Bailey, S.R., Erbel, R., Fitzgerald, P.J., Pinto, F.J., Rosenfield, K., Siegel, R.J., Tuzcu, E.M., Yock, P.G.: American College of Cardiology Clinical Expert Consensus Document on Standards for Acquisition, Measurement and Reporting of Intravascular Ultrasound Studies (IVUS). J Am Coll Cardiol, vol. 37 (5), pages 1478-1492, 2001.

13. Mintz, G.S., Painter, J.A., Pichard, A.D., Kent, K.M., Satler, L.F., Popma, J.J., Chuang, Y.C., Bucher, T.A., Sokolowicz, L.E., Leon, M.B.: Atherosclerosis in Angiographically " Normal " Coronary Artery Reference Segments: an Intravascular Ultrasound Study with Clinical Correlations. J Am Coll Cardiol, vol. 25 (7), pages 1479-1485, 1995.

14. Mojsilovic, A., Popovic, M., Amodaj, N., Babic, R., Ostojic, M.: Automatic Segmentation of Intravascular Ultrasound Images: A Texture-Based Approach. Ann Biomed Eng, vol. 25 (6), pages 1059-1071, 1997.

15. Osher, S., Sethian, J.A.: Fronts Propagating with Curvature-Dependent Speed: Algorithms Based on Hamilton-Jacobi Formulations. J Comput Phys, 79, pages 12-49, 1988.

16. Sethian, J.A.: Level Set and Fast Marching Methods. Evolving Interfaces in Computational Geometry, Fluids Mechanics, Computer Vision and Materials Science. 2e ed. Cambridge, UK, Cambridge University press, 1999.

17. Sethian, J.A.: A fast marching level set method for monotonically advancing fronts. Proceedings of the National Academy of Sciences of the United States of America, vol. 93, pages 1591-1595, 1996.

18. Sifakis, E., Garcia, C., Tziritas, G.: Bayesian Level Sets for Image Segmentation. $\mathrm{J}$ Vis Commun Image R, vol. 13, pages 44-64, 2002.

19. Sonka, M., Zhang, X., Siebes, M., Bissing, M.S., DeJong, S.C., Collins, S.M., McKay, C.R.: Segmentation of Intravascular Ultrasound Images: A KnowledgeBased Approach. IEEE T Med Imaging, vol. 14 (4), pages 719-732, 1995. 